\title{
ON THE SLEEP RESPONSE INDUCED ELECTRICALLY IN CATS
}

\author{
YOSHIHIKO IWASE AND KATSUHIKO YANAZUME* \\ Research Institute of Applied Electricity, Hokkaido University
}

For many years, various attempts have been made to induce the sleep state, by electrical stimulation of the brain and it has been proved to have many obvious advantages over chemical narcosis. In order to produce such an electronarcosis, electrical parameters (1), especially the shape and frequency of stimulating current have been taken into consideration.

Here the total energy and the stimulation period should be one of the indispensable conditions. Attention was therefore paid to the effects of prolonged stimulation by the alternating and pulse currents, and this method was introduced as a new type of electronarcosis (2). The present experiment was carried out in the attempt to induce sleep responses by "monopolar" stimulation applied to the head.

\section{METHOD}

Unanesthetized cats were used. Stimulations were provided by an alternating current and rectangular pulses ( 0.5 to $10 \mathrm{msec}$.), at a constant frequency of 50 cycles/sec. Either hemisphere of the brain was stimulated monopolarly through the silver electrodes arranged to the frontal cortex and the eye's cornea. In early experiments of inducing sleep, the continous stimulation method with gradually rising voltage was employed. The later improved method, developed originally by Hess (3), was to use intermittent stimulation in which sleep was induced with less difficulty. Usually, several stimulations of 1-2 min. periods were required to induce sleep responses. The interval ranged from 0.5 to 1 min. or more depending on the behavior of animals, especially respiration.

In all cases, the stimuli were started at low voltage, and were gradually increased. The sleep response was to appear with a definite strength of stimuli. Voltages and currents necessary to induce sleep were determined by a calibrated oscilloscope. Using the vacum-tube voltmeter, the potential gradients during stimulation were mapped at forty points of the subcortical levels.

\section{RESULTS AND DISCUSSION}

Four types of responses resulted from electrical stimulation-motor, adynamia, depressive and sleep responses, the latter two of which resembled closely those described in detail by Hess. The motor and adynamia responses were usually produced by continous stimulation. The motor response was of a less

Received for publication October 13, 1955.

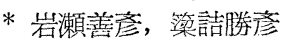


quiet nature, and the adynamia was a motionless state characterized by the autonomic activity.

In another series of experiments, in which intermittent stimulation was employed. sleep and depressive responses, showing a striking similarity in posture to one another, were more readily elicited. The animal, at first awake, became sleepy a few minutes after the onset of stimulation. However, in these experiments sleep could not last long, seemingly because it was disturbed chiefly by acoustic stimulations as is the case with normal sleep. On the contrary, the depressive response could not be reversed by any means. The sleep response was selectively observed with adequate stimulations, such as using a first low and gradually rising voltage, with well controlled intervals. If the stimulation continued longer, the sleep response tended more and more to the depressive or motor response. When the strength of stimulation was increased rapidly, a circling movement with forced turning of the head was inevitably produced.

The voltages necessary to induce sleep were determined using changes in the behavior of the animal and autonomic responses, such as respiration, pulse, and reflex of pupils as indices. Pulse currents of 0.5 to $5.0 \mathrm{msec}$. were usually employed and it was compared with the alternating current. With the stimulation of pulse current of $5.0 \mathrm{msec}$., the maximum of threshold voltages needed to induce sleep response was $2 \mathrm{~V}$., which was the same as required in the case of the alternating current (fig. 1). This pulse was adequate for inducing sleep, because of no production of other responses. In the case of the pulse current of $0.5 \mathrm{msec}$., distinct differences were observed in both the voltages and the stimulation periods. In these experiments, however, the nature of the sleep response was not changed. Nevertheless, such findings brought forth the information that the sleep response could be induced by gradually increasing intermittent stimulations of both alternating and rectangular pulse currents. This conception is a challenge to earlier works along this line.

Consideration must be now directed to the nature of the threshold and the stimulation periods for inducing sleep. Lilly (4) demonstrated changes in threshold (forelimb movement) with train duration at various frequencies. Within the range of train duration of from 0.1 to 20.0 sec., there was a uniform fall in threshold with increasing train duration. However, a dramatical threshold increase due to longer stimulation periods (10 to 60 min.) was demonstrated by Suzuki (5). Thus, there must be some reason to believe that the threshold value is a function of the length of

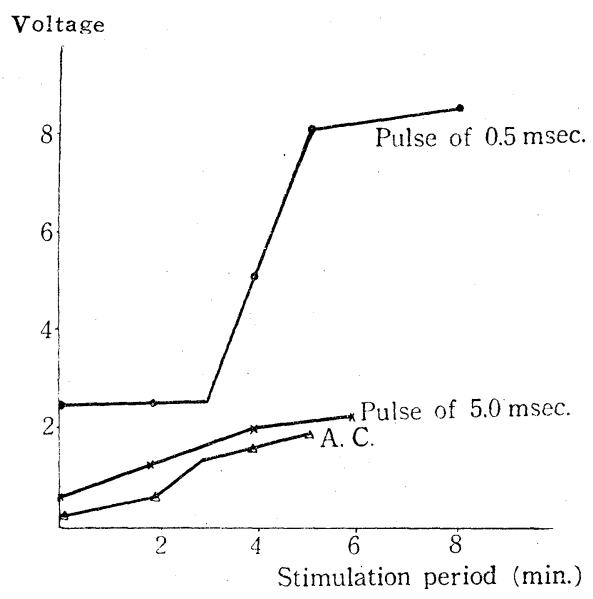

FIG. 1. Relations between threshold voltages and the length of stimulation periods necessary to induce sleep. 
stimulation period. Indeed, the present results showed that longer periods of stimulation ( 5 to $8 \mathrm{~min}$.) raised the threshold of stimulation and consequently stronger current was required for inducing sleep response. In other words, a stronger stimulation could be applied to the brain without excitation.

Potential gradients established between the electrodes have been mapped in detail at the subcortical levels (6). The experimental observations correspond fairly well with neuronal arrangement in the brain, that is to say, nervous structure such as "corpus callosum" alters the electrical field and produces a specific distribution of potential gradients. From the map (fig. 2), it is recognized that the potential gradient established around the hypothalamus is $0.3 \mathrm{~V} / 5$ $\mathrm{mm}$. at the vertical axis and 10-fold greater than that of the cortex. It must be emphasized that the high level of excitation represented by the steeper potential gradient can be maintained around the anterior sub- and hypothalamus in the monopolar stimulation.
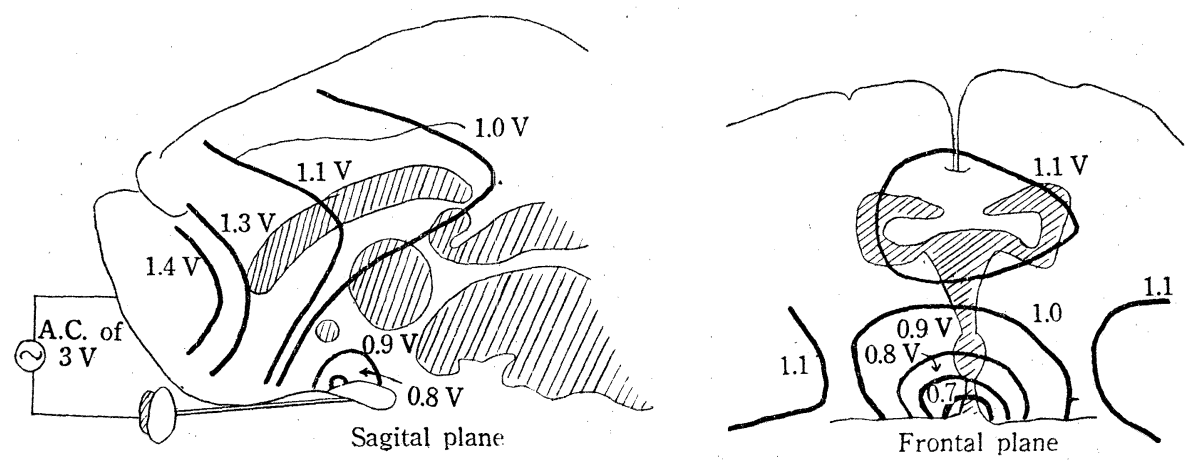

FIG. 2. Potential gradients mapped in the cat's brain during the flow of alternating current.

From studies on spindle bursts (7), hypothalamic stimulation (8), and afterdischarges (9), the nervous system for inducing sleep was proved to concern the subcortical system. In addition, the mapping of potential gradients proposed new information that the excitement around the sub- and hypothalamus was most necessary for the onset of sleep. No doubt, this excitement would be widely radiated extending over the whole mass of the brain in accordance with the process of falling asleep.

Regrettably, the behavior of animals did not help to distinguish sleep clearly from depressive response. Special attention must be paid to the exact recognition of sleep and depressive responses.

(1) Sleep response has been differentially studied by the characteristic type of the respiration curve. It has been shown in general that the mode of stimulation can be directly correlated with definite respiratory changes (fig. 3). Respiration so observed, is thus an indirect measure of brain activity. This is by no means the only method which can be used to differentiate the responses, nor the most adequate, but the simplest one.

The urgent necessity of an objective method for recording brain activities has been apparently satisfied in part by recording the brain potential and the 
FIG. 3. The influences of electrical stimulation upon the respiration in induced sleep and depressive responses.

Sleep response was accompanied by some decrease in rate and depth of respiration observed several min. after the onest of stimulation (solid line). In depressive response, the respiratory depth increased shortly after the begining of stimulation (broken line). Shaded areas at the bottom indicate the durations of depressive and sleep responses. After a short initial period of stimulation, the type of respiration was, on the contrary, rapidly increased in depresive response. Broken and solid lines show respectively depressive and sleep responses occured corresponding with the marks on the abscissa.

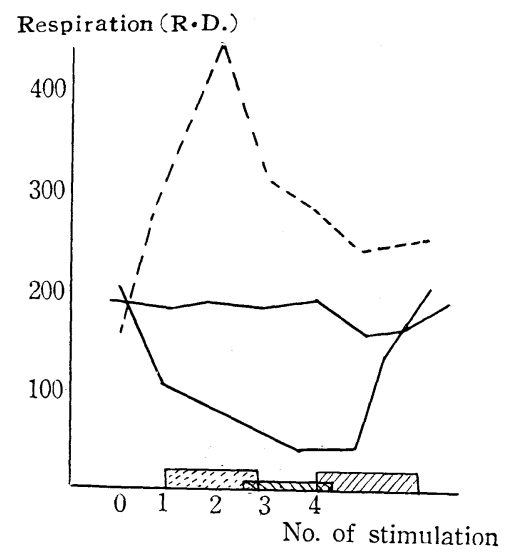

steady potential of brain.

(2) The brain potential.

Stimulation in cats can also induce reversible brain potentials as recently demonstrated by Hess (10). Spindle bursts activity of high frequency, suggesting drowsiness, and $\delta$ waves recorded during the stimulation appeared rapidly, but not in a definite sequence as observed in human subjects (Atlas 1). In depressive response, $\alpha$ waves which characterized the resting state disappeared completely. Frequently the changes in behavior were followed by alternations of brain potentials during the sleep. One must note, however, the brain potential does not always correspond to that which is usually associated with the behavior of the animal. Similar conclusions were reached by Hess that at the onset of induced sleep certain discrepancies occurred.

(3) The steady potential of brain (11).

The steady potential in the sensory-motor cortex was positive with reference to the sciatic nerve in all instances, and the potential ranging from 2 to $7 \mathrm{mV}$ in any given animal showed small variations in the resting state. The induced sleep always produced significant changes consisting of a decreasing deflection, while adynamia and motor responses produced an increasing one. This finding reflects either extreme of change of steady potential obtained in the depressed activity of cortical cells (Goldensohn (12)).

The advent of these techniques provided the impetus for differentiating true sleep from other depressed states. Valuable contributions to resolving this problem have been made by Hunter and Yoshii. Each of responses, such as those carried out by Hunter (13), was independently produced only by varying the parameters of the stimulation. According to Yoshii (14), the differentiation between sleep and seizure was displayed by the measurement of electrical impedance of the brain. He demonstrated a definite relationship between brain impedance and depth of electronarcosis. As the electronarcosis became deeper, the brain impedance increased gradually and was stabilized at a high level.

It is considered that the significance of such procedures lies in differentiating sleep and other responses. They could, however, make no consideration as to whether or not the depressive response was actually in the sleeping state. 
After all, the authors have reached to the resolution of some of the practical trials regarding the effects of stimulation.

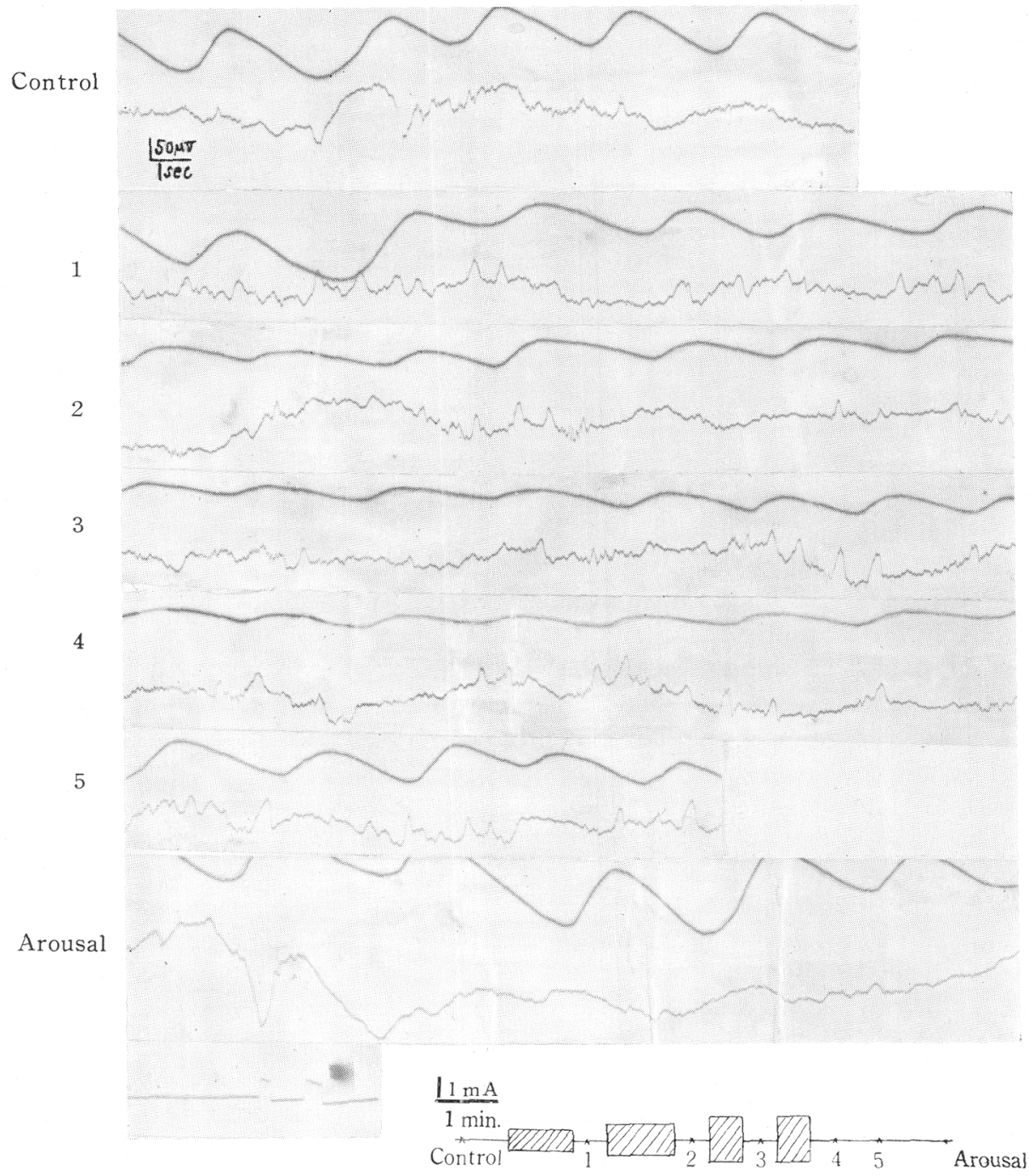

ATLAS 1. Electrical activity of the frontal cortex and respiratory change during the onest and duration of sleep induced by the electrical stimulation of alternating currents.

The cortical activity was registered from the frontal cortex using $\mathrm{Ag}-\mathrm{AgCl}$ electrodes ( $2 \mathrm{~mm}$. diameter) placed on the dura mater through holes in the skull, in monopolar disposition.

Throughout duration of sleep experiments, the EEG was recorded except during periods of stimulation. 


\section{SUMMARY}

The sleep response was induced by the monopolar stimulation applied between the frontal cortex and the eye cornea in cats.

The results were as follows:

1. Sleep response was induced by the prolonged stimulation of alternating and rectangular pulse currents of $50 \mathrm{cycles} / \mathrm{sec}$. The voltages necessary to induce sleep were compared with the alternating and pulse currents at the range of 0.5 to $10 \mathrm{msec}$. Especially, the pulse current of $5 \mathrm{msec}$. was adequate for inducing sleep, because of no production of other responses.

2. The steep potential gradient was mapped around the sub- and hypothalamus by monopolar stimulation.

3. The sleep and depressive responses have been differentiated by respiratory and electrophysiological characteristics.

The authors wish to express their appreciation to Professor T. Minoshima who contributed many suggestions throught the course of the research.

This research was assisted by a grant from the Ministry of Education of Japan.

\section{REFERENCES}

1. IWASE, Y. Electrophysiological problems on induced sleep. Monograph Series of Res. Inst. Appl. Elec., Sapporo No. 5, 1955.

2. IWASE, Y. AND YANAZUME, K. Studies on sleep induced by electric stimulation (Part II). Bull. Res. Inst. Appl. Elec. 5: 71, 1953 (Japanese).

3. AKert, K., KOELla, W. P. AND HESS, W. R. Sleep produced by electrical stimulation of the thalamus. Am. J. Physiol. 168: 260, 1952.

4. Lilly, J. C., Austin, G. M. AND Chambers, W. W. Threshold movements produced by excitation of cerebral cortex and efferent fibres with some parametric regions of rectangular current pulses (Cats and monkeys). J. Neurophysiol. 16: 319, 1952.

5. Suzuki, M. Pri la efikoj de elektrodoj de elektra trafluigado. J. Physiol. Soc. Jap. 17: 223, 1955.

6. NAGAI, S. Studies on the potential gradient in the brain during the passage of electric current (Studies on induced sleep, Part III). Bull. Res. Inst. Appl. Elec. 6: 29, 1954 (Japanese).

7. Yanazume, K., TAZaki, A., NaKamura, T. AND Hashimoto, N. On the spindle bursts activity of frontal cortex by electric stimulation (Studies on induced sleep, Part III). Bull. Res. Inst. Appl. Elec. 6: 21, 1954 (Japanese).

8. IWASE, Y. The effect of hypothalamic stimulation on hypothalamic and cortical potentials (Studies on induced sleep, Part IV). Bull. Res. Inst. Appl. Elec. 6: 103, 1954 (Japanese).

9. YANAZUME, K. Unpublished.

10. Hess, W. R., Koella, W. P. AND AKert, K. Cortical and subcortical recording in natural and artificially induced sleep in cats. EEG. Clin. Neurophysiol. 5: 75, 1953.

11. OIKAWA, I. Studies on the steady potential of brain. Nō to Shinkey (Brain and nerve) in press (Japanese).

12. Goldensohn, E. S., Schoenfeld, R. L. And Hoefer, P. F. A. The slowly changing voltage of the brain and the electrocorticogram. EEG. Clin. Neurophysiol. 3: 231, 1951.

13. HUNTER, J. AND JASPER, H. H. Effect of thalamic stimulation in unanesthetized animal. EEG. Clin. Neurophysiol. 1: 305, 1949.

14. Yoshir, N. Physiology of the epilepsy. Nö to Shinkey (Brain and nerve). 5: 57, 1953 (Japanese). 